\title{
Millimeter-Wave Lumped Element Superconducting Bandpass Filters for Multi-Color Imaging
}

\author{
Shwetank Kumar, Anastasios Vayonakis, Henry G. LeDuc, Peter K. Day, Sunil Golwala, and \\ Jonas Zmuidzinas, Member, IEEE
}

\begin{abstract}
The opacity due to water vapor in the Earth's atmosphere obscures portions of the sub-THz spectrum $(\mathrm{mm} / \mathrm{sub}-\mathrm{mm}$ wavelengths) to ground based astronomical observation. For maximum sensitivity, instruments operating at these wavelengths must be designed to have spectral responses that match the available windows in the atmospheric transmission that occur in between the strong water absorption lines. Traditionally, the spectral response of $\mathbf{m m} / \mathbf{s u b}-\mathbf{m m}$ instruments has been set using optical, metal-mesh bandpass filters [1]. An alternative method for defining the passbands, available when using superconducting detectors coupled with planar antennas, is to use on-chip, superconducting filters [2]. This paper presents the design and testing of superconducting, lumped element, on-chip bandpass filters (BPFs), placed inline with the microstrip connecting the antenna and the detector, covering the frequency range from $209-416 \mathrm{GHz}$. Four filters were designed with pass bands 209-274 GHz, 265-315 GHz, 335-361 GHz and 397-416 GHz corresponding to the atmospheric transmission windows. Fourier transform spectroscopy was used to verify that the spectral response of the BPFs is well predicted by the computer simulations. Two-color operation of the pixels was demonstrated by connecting two detectors to a single broadband antenna through two BPFs. Scalability of the design to multiple (four) colors is discussed.
\end{abstract}

Index Terms-Bandpass filters, passive microwave circuits, submillimeter astronomy.

\section{INTRODUCTION}

$\mathbf{T}$ HERE are various existing designs for defining the passbands of $\mathrm{mm} / \mathrm{sub}-\mathrm{mm}$ astronomical cameras. Metal-mesh filters [1] have been used in conjunction with Infrared (IR) blocking materials such as teflon and fluorogold to make quasi-optical filters. This approach, however, has the disadvantage of preventing multi-color pixel operation, since the entire pixel array is irradiated by the same frequency band. Multicolor pixel operation is desirable for upcoming camera designs [3]

Manuscript received August 19, 2008. First published May 27, 2009; current version published July 10, 2009. This work was supported in part by the NASA Science Mission Directorate, Jet Propulsion Laboratory, and the Gordon and Betty Moore Foundation.

S. Kumar is with IBM T.J. Watson Research Center, Yorktown Heights, NY 10598 USA (e-mail: shwetank@us.ibm.com). He was with the Department of Applied Physics, California Institute of Technology Pasadena, CA 91125 USA.

A. Vayonakis, S. Golwala, and J. Zmuidzinas are with the Department of Physics, California Institute of Technology, Pasadena, CA 91125 USA (e-mail: avayona@submm.caltech.edu; golwala@ caltech.edu; jonas@caltech.edu).

H. G. LeDuc and P. K. Day are with the Jet Propulsion Laboratory, Pasadena, CA 91109 USA(e-mail: henry.g.leduc@jpl.nasa.gov; peter.k.day@jpl.nasa.gov).

Color versions of one or more of the figures in this paper are available online at http://ieeexplore.iee.org.

Digital Object Identifier 10.1109/TASC.2009.2017884 to increase the overall throughput for a wide field survey by capturing more photons in different frequency bands. This has many advantages [4], including more efficient use of the focal plane. Further, the quasi-optical mesh filters are typically designed for normal-incidence operation; radiation incident at oblique angles may leak through and load the detector. Proper operation of quasioptical filters also requires care and attention to detail. For instance, the filters need to be carefully heat sunk in order to prevent detector loading due to radiation emitted by the filters themselves. These issues may be addressed using on-chip designs for the filters. Although we chose to use lumped-element designs, on-chip bandpass filters may also be implemented using distributed circuit elements [5]. However, using distributed circuit elements causes the bandpass filters to leak at higher harmonic frequencies requiring a cascaded lowpass filter. This adds to the complexity of the circuit and takes up extra real estate on the chip. The lowpass filter may instead be implemented using a metal mesh filter as long as the issues mentioned above are addressed. The lumped-element, on-chip bandpass filters presented in this paper offer a relatively simple and compact solution to the problem of multicolor $\mathrm{mm} / \mathrm{sub}-\mathrm{mm}$ imaging. We will now describe the detailed filter design and the measurement results.

\section{BANDPASS FILTER DESIGN}

\section{A. Lumped-Element Circuit Design}

We use a three-element Butterworth lowpass filter prototype [Fig. 1(a)] with cutoff at $\omega_{c}=1$ and terminated in a $1 \Omega$ load as a basis for design of our bandpass filters. This normalized lowpass filter has element values $L_{n}=1, C_{n}=2$ [Fig. 1(a)]. It is converted to a lumped-element bandpass filter using the usual transformations [6]: replacing the series inductor by a series combination of inductor $\left(L_{1}=Z_{0} L_{n} / \omega_{0} \Delta\right)$ and capacitor $\left(C_{1}=\Delta / Z_{0} L_{n} \omega_{0}\right)$, and shunt capacitor by a parallel combination of inductor $\left(L_{2}=Z_{0} \Delta / C_{n} \omega_{0}\right)$ and capacitor $\left(C_{2}=C_{n} / Z_{0} \Delta \omega_{0}\right)$ [Fig. 1(b)]. $Z_{0}$ is the characteristic impedance of the filter, chosen to be equal to the characteristic impedance of the microstrip transmission line which brings submillimeter power from the antenna and is equal to $20 \Omega$. Here $\Delta=\omega_{c 2}-\omega_{c 1}$ is the design bandwidth of the BPF and $\omega_{0}=\sqrt{\omega_{c 1} \omega_{c 2}}$ is the center frequency given by the geometric mean of the $3 \mathrm{~dB}$ cutoff frequencies of the BPF. $\omega_{c 1}$ and $\omega_{c 2}$ are the upper and lower cutoff frequencies of the bandpass filter, respectively. For the ease of fabrication of the circuit, shunt inductors to ground should be avoided. For sufficiently narrow bandwidth (nominally 10-20\%), impedance inverters can be used to replace a parallel shunt admittance $\left(Y_{p}\right)$ 
a)
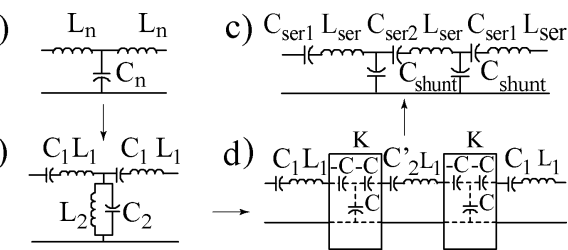

Fig. 1. Steps for lumped-element bandpass filter circuit design. (a) 3-pole Butterworth low-pass filter prototype. $L_{n}$ and $C_{n}$ are normalized inductor and capacitor, respectively. (b) Bandpass filter with series LC and shunt parallel LC resulting from the prototype low-pass filter. (c) Bandpass filter with impedance inverter blocks and no shunt inductors. (d) Final lumped-element bandpass filter circuit with no shunt inductors.

TABLE 1

Circuit Element VAlues for Filters in Fig. 1(D) With PASSBAND DEFINED BY ATMOSPHERIC TRANSMISSION WINDOWS

\begin{tabular}{ccccc}
\hline & BPF1 & BPF2 & BPF3 & BPF4 \\
Element & $209-265$ & $274-315$ & $335-361$ & $396-416$ \\
Values & $\mathrm{GHz}$ & $\mathrm{GHz}$ & $\mathrm{GHz}$ & $\mathrm{GHz}$ \\
\hline$L_{\text {ser }}(\mathrm{pH})$ & 75.00 & 123.2 & 146.91 & 201.04 \\
$C_{\text {ser } 1}(\mathrm{fF})$ & 7.37 & 2.48 & 1.50 & 0.79 \\
$C_{\text {ser } 2}(\mathrm{fF})$ & 9.01 & 2.69 & 1.59 & 0.82 \\
$C_{\text {shunt }}(\mathrm{fF})$ & 40.23 & 31.36 & 26.97 & 23.10 \\
\hline
\end{tabular}

by a series impedance $\left(Z_{s}\right)$ for two-port networks [7] [Fig. 1(c)]. Using the identity $Z_{s}=K^{2} Y_{p}$, where $Z_{s}=j \omega L_{1}+\left(1 / j \omega C_{2}^{\prime}\right)$, $Y_{p}=j \omega C_{2}+\left(1 / j \omega L_{2}\right)$, and $K$ is the impedance inverter, we get the values for $K$ and series capacitance $C_{2}^{\prime}$ using $K^{2}=$ $L_{1} / C_{2}$ and $C_{2}^{\prime}=L_{2} C_{2} / L_{1}$. The impedance inverter is implemented using a two-port $\mathrm{T}$ network with the series branches containing capacitors $-C$ and shunt branch containing capacitor $C$ where $C=1 / \omega_{0} K$ [Fig. 1(c)]. The negative capacitance in the series branches can be absorbed into $C_{1}$ and $C_{2}^{\prime}$ to give the final circuit [Fig. 1(d)]. Here $L_{\text {ser }}=L_{1}, C_{\text {shunt }}=C$, $C_{\text {ser } 1}=C C_{1} /\left(C-C_{1}\right)$, and $C_{\text {ser } 2}=C C_{2}^{\prime} /\left(C-2 C_{2}^{\prime}\right)$. The lumped circuit element values derived using this synthesis method are further optimized using Agilent ADS [8], a circuit simulation software package, to give a power transmission of at most $-40 \mathrm{~dB}$ in stop band, unity transmission in passband with sharpest possible rolloff. These optimized values for various lumped components for the four bandpass filters are listed in Table I.

\section{B. Circuit Layout and Fabrication}

The schematic of the filter layout is shown in Fig. 2. The integrated inline BPF circuit was fabricated on chip with the other pixel components using photolithography. The inductors were implemented using spiral geometry and the capacitors in the parallel plate configuration. The bottom layer is $200 \mathrm{~nm}$ thick niobium film deposited on the silicon substrate using DC magnetron sputtering. The ground plane, spiral inductors (Fig. 2 shown in dark grey) and lower plate of the parallel plate capacitors [not visible in Fig. 2(a)] are patterned on this layer using photoresist and $\mathrm{SF}_{6}$ Inductively Coupled Plasma (ICP) etching. Following this, a $400 \mathrm{~nm}$ thick $\mathrm{SiO}_{2}$ dielectric layer is deposited on the bottom layer by RF magnetron sputtering with substrate bias. Subsequently, a $200 \mathrm{~nm}$ thick layer of niobium is deposited using DC $\backslash$ magnetron sputtering and patterned using a $\mathrm{BCl}_{3} / \mathrm{Cl}_{2}$ inductively coupled plasma (ICP) etch to

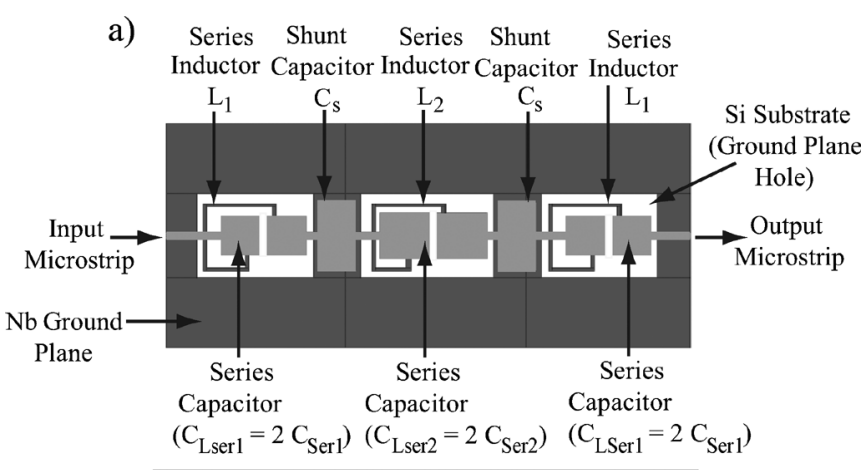

b)

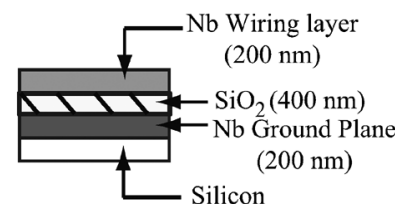

Fig. 2. SONNET layout for the bandpass filters: (a) top view, (b) side view. The top microstrip is made of niobium (light grey). Below it is a layer of $\mathrm{SiO}_{2}$ (white hatched with black lines, not visible in the top view), then niobium ground plane (dark grey). The series inductors and capacitors are fabricated on top of the silicon substrate (white) directly in holes in the ground plane.

form the wiring layer of microstrip geometry (Fig. 2, shown in light grey). The dielectric is then removed using a $\mathrm{CHF}_{3} / \mathrm{O}_{2}$ ICP etch. The input microstrip brings the broadband submillimeter power from the slot antenna to the filter (Fig. 8). In order to avoid having to make conducting via contacts between the bottom niobium layer and the top niobium layer, the first series capacitor is divided into two series capacitors each having double the design capacitance $\left(C_{L S e r 1}=2 C_{s e r 1}\right)$ and placed on either side of the spiral inductor as shown in Fig. 2(a). A very short microstrip line connects the second series capacitor to the shunt capacitor. This design is then repeated for the remaining two series capacitor-inductor sections. Finally, the output microstrip takes the band-limited submillimeter power to the detector. For our device the photon detector was implemented using a kinetic inductance detector which is a pair-breaking superconducting detector [9], [16], [17], that for the purposes of this design will behave like an impedance matched attenuator at the end of the filter output microstrip.

\section{Component Design}

1) Spiral Inductor Design: Correct design of the inductor is critical for the optimal performance of the bandpass filter. The inductor may either be implemented using the coplanar waveguide (CPW) geometry which is simpler to design but can only provide relatively small inductance values, or a spiral geometry, which was finally used for our design. To make the design choice we calculated the length of CPW required to provide the minimum required inductance of $75 \mathrm{pH}$, as follows. Let $Z_{0}(=20 \Omega), Z_{c}(=50 \Omega), \varepsilon_{d}(=10.5), f(=250 \mathrm{GHz})$ be the characteristic embedding impedance of the external circuit, characterstic impedance of CPW used to implement the lumped inductor, substrate (sapphire) dielectric constant and the filter center frequency, respectively. The effective dielectric constant for CPW $\varepsilon_{\text {eff }}=\left(\varepsilon_{d}+1\right) / 2=5.75$. This may be calculated more accurately using SONNET simulations. Using equations 


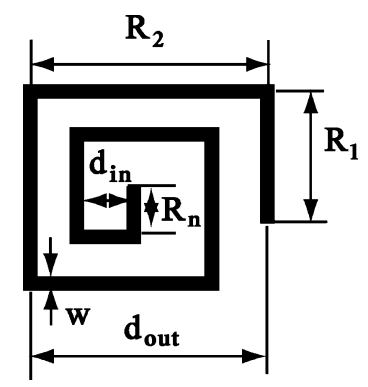

Fig. 3. Spiral inductor schematic detailing the dimensions used in the inductor design. $R_{n}$ is the length of the $n$th arm of the spiral inductor with $n=1$ for the outermost arm. $d_{\text {out }}$ and $d_{i n}$ are the outermost and innermost dimension of the inductor, respectively. $w$ is the trace width.

for transmission line characteristic impedance and propagation constant we can get the inductance per unit length:

$$
\mathcal{L}=\frac{\beta Z_{c}}{\omega}=\frac{\sqrt{\varepsilon_{e f f}} Z_{c}}{c}
$$

where $c$ is the speed of light, $\beta$ is the propagation constant, and $\omega$ is the frequency in radians/sec. The CPW propagation wavelength at $250 \mathrm{GHz}$ is $500 \mu \mathrm{m}$. Using equation (1) the length of CPW line required to implement inductance $(L=75 \mathrm{pH})$ lumped inductor is $l=L c / \sqrt{\varepsilon_{\text {eff }}} Z_{c}=187.67 \mu \mathrm{m}$, which is a significant fraction of the CPW wavelength. Hence, a $75 \mathrm{pH}$ inductor implemented using CPW with the above $Z_{c}$ will behave like a distributed element. This will cause the filter to leak at integral multiples of the transmission line fundamental mode. A spiral geometry for the inductor can instead be used to provide the high values of the inductance needed for the bandpass filters. The rectangular spiral (Fig. 3) was chosen for layout convenience and the initial guess for the geometry was determined by calculating the inductance using the formulae available in the literature [9]:

$$
L=\frac{\mu n^{2} d_{a v g} c_{1}}{2}\left(\ln \left(c_{2} / \rho\right)+c_{3} \rho+c_{4} \rho^{2}\right)
$$

Here $\rho=\left(d_{\text {out }}-d_{\text {in }}\right) /\left(d_{\text {out }}+d_{\text {in }}\right), d_{\text {avg }}=\left(d_{\text {out }}+d_{\text {in }}\right) / 2$, $L$ is the inductance and $c_{1}, c_{2}, c_{3}$, and $c_{4}$ are geometry dependent coefficients with values $1.27,2.07,0.18$, and 0.13 , respectively, for rectangular spiral geometry. $\rho$ is the filling fraction, with $d_{\text {in }}$ and $d_{\text {out }}$ the innermost and the outermost dimensions of the inductor (Fig. 3), $\mu$ is the magnetic permeability, and $n$ is the number of turns. This formula is not very accurate for large values of $s / w$ and the final inductor geometry is obtained by laying it out in SONNET [10], simulating its impedance, and re-optimizing the geometry to give the desired value. The exact final dimensions of each of the inductors finally used to implement the BPFs are tabulated in Table II.

2) Capacitor Design: The dimensions for the capacitor with capacitance values tabulated in Table I can be estimated using parallel plate formula $C=\varepsilon_{0} \varepsilon_{d} A / d$, where $\varepsilon_{0}$ is the free space permittivity, $\varepsilon_{d}(=4)$ is the dielectric constant of $\mathrm{SiO}_{2}, A$ is area of the parallel plates, and $d$ is the thickness of $\mathrm{SiO}_{2}$. The capacitor dimensions were further optimized by laying out capacitor-inductor series combinations in SONNET and optimizing
TABLE 2

SPIRAL INDUCTOR DIMENSIONS IN $\mu \mathrm{m}$ FOR SCHEMATIC IN FIG. 3

\begin{tabular}{ccccccccc}
\hline & \multicolumn{2}{c}{ BPF1 } & \multicolumn{2}{c}{ BPF2 } & \multicolumn{2}{c}{ BPF3 } & \multicolumn{2}{c}{ BPF4 } \\
& $L_{1}$ & $L_{2}$ & $L_{1}$ & $L_{2}$ & $L_{1}$ & $L_{2}$ & $L_{1}$ & $L_{2}$ \\
\hline$R_{1}$ & 3.5 & 2.5 & 5.5 & 5.0 & 7.0 & 7.0 & 7.5 & 7.5 \\
$R_{2}$ & 19.5 & 20.0 & 17.5 & 17.5 & 15.5 & 15.5 & 15.5 & 15.5 \\
$R_{3}$ & 21.0 & 21.0 & 21.0 & 21.0 & 17.0 & 17.0 & 16.5 & 16.0 \\
$R_{4}$ & 12.0 & 10.0 & 14.5 & 14.5 & 12.5 & 12.5 & 12.5 & 12.5 \\
$R_{5}$ & 4.5 & 2.5 & 8.0 & 8.5 & 13.0 & 13.0 & 12.5 & 12.0 \\
$R_{6}$ & 0 & 0 & 2.5 & 2.5 & 5.0 & 5.5 & 9.5 & 9.5 \\
$R_{7}$ & 0 & 0 & 0 & 0 & 3.0 & 3.0 & 8.0 & 8.0 \\
$R_{8}$ & 0 & 0 & 0 & 0 & 0 & 0 & 3.5 & 3.0 \\
$t$ & 1.0 & 1.0 & 1.0 & 1.0 & 1.0 & 1.0 & 1.0 & 1.0 \\
\hline
\end{tabular}

TABLE 3

DIMENSIONS OF CAPACITORS IN FIG. 2(A) IN $\mu \mathrm{m}$

\begin{tabular}{lcccccc}
\hline & \multicolumn{3}{c}{ BPF1 } & & \multicolumn{3}{c}{ BPF2 } \\
& $C_{\text {Lser1 }}$ & $C_{\text {Lser2 } 2}$ & $C_{\text {shunt }}$ & $C_{\text {Lser1 }}$ & $C_{\text {Lser2 }}$ & $C_{\text {shunt }}$ \\
\hline Length & 11.5 & 14.5 & 10.5 & 8.0 & 5.0 & 10.5 \\
Width & 13.0 & 15.0 & 23.0 & 9.0 & 7.5 & 19.5 \\
\hline \hline & & BPF3 & & & BPF4 & \\
& $C_{\text {Lser1 }}$ & $C_{\text {Lser } 2}$ & $C_{\text {shunt }}$ & $C_{\text {Lser } 1}$ & $C_{\text {Lser } 2}$ & $C_{\text {shunt }}$ \\
\hline Length & 7.0 & 4.5 & 11.5 & 3.5 & 4.5 & 10.0 \\
Width & 6.0 & 7.5 & 17.5 & 5.0 & 5.0 & 10.0 \\
\hline
\end{tabular}

the dimensions of the layout so that it has a resonance at the same frequency as the series combination of lumped $L C$ with values in the Table I. The final dimensions of the capacitors for each of the filters are tabulated in Table III. Thickness and dielectric constant of $\mathrm{SiO}_{2}$ is $4000 \AA$ and $\varepsilon_{d}=4$, respectively. The dimensions of the parallel plate capacitors used to implement first and third series capacitor in Fig. 1(d) are exactly the same. Length direction is assumed to be the direction of propagation of submillimeter radiation.

3) SONNET Simulations: The response of each of the circuit elements, and subsequently the entire layout, was simulated using SONNET [10] - a planar 3D electromagnetic simulator. The SONNET layout of the BPF is seen in Fig. 2. Initial simulations assumed the niobium ground plane and wiring layers (seen in dark and light grey, respectively) to be perfectly conducting 2D sheets (zero thickness) with $400 \mathrm{~nm} \mathrm{SiO}_{2}$ dielectric between them. The lumped-element geometries were optimized to produce the required bandpass filter response.

\section{Effect of Superconductivity on the Filter Response}

Niobium, which is used to fabricate the bandpass filters, superconducts at the pixel operating temperatures (niobium $T_{c}=$ $9.2 \mathrm{~K}, \mathrm{~T}_{o p}=220 \mathrm{mK}$ ). Superconductors have a finite surface impedance at microwave and submillimeter wavelengths [11], and simulating them as 2D planar films of perfect conductors can lead to significant errors in the bandpass filter response calculation. SONNET can be used to accurately simulate the bandpass filter response by incorporating the effects of superconductivity and finite conductor thickness. This can be done by replacing a single perfectly conducting zero thickness planar sheet with multiple zero thickness planar sheets of a given surface impedance $\left(Z_{x}\right)$, different from the actual surface impedance $\left(Z_{s}\right)$ of the superconducting film, as a model for a thick superconducting film [12], [13]. For $\lambda_{L} \simeq t$, where $\lambda_{L}$ is the London penetration depth and $t$ the superconductor film thickness, a two-sheet model is sufficient [13]. In the local limit, 


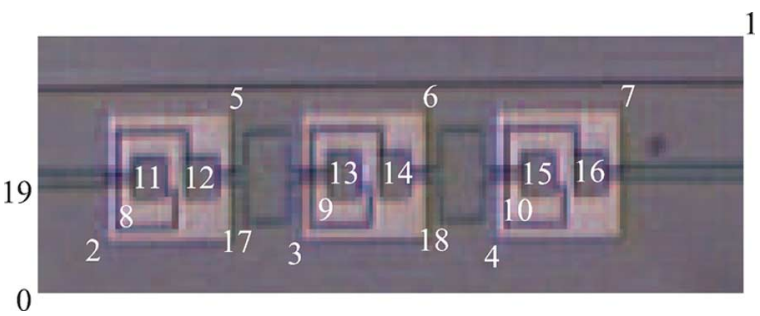

Fig. 4. Microscope photograph of bandpass filter. The hole in the niobium ground plane showing the exposed substrate is colored in a lighter shade of grey. The niobium ground plane and the wiring layer are seen in dark grey. Numbers indicate points on the image as follows: 0 is the origin, 1 the upper-right corner of the layout. 2, 3, 4 and 5, 6, 7 are the lower-left and upper-right corners, respectively, of the holes in the niobium ground plane. $8,9,10$ are the lower-left corners of the spiral inductor. 11,12,13,14, 15, 16 are the lower-left corners of series capacitors $C_{\text {Lseries1,2,3 }} .17,18$ are the lower-left corners of the two shunt capacitors. 19 is the lower-left corner of the input microstrip. The microstrip width is $2.5 \mu \mathrm{m}$.

at temperature well below $T_{c}$ and frequency well below the gap frequency, assuming $Z_{\eta}(=377 \sim \Omega) \gg \omega \mu_{0} \lambda_{L}(=0.14 \Omega)$ where $Z_{\eta}$ is the free space impedance, and $\mu_{0}$ is free space permeability, the surface impedance of the superconducting film is given by [13]:

$$
Z_{s}=j \omega \mu_{0} \lambda_{L} \operatorname{coth}\left(t / \lambda_{L}\right)
$$

The impedance seen by a TEM mode traveling along a transmission line is essentially the same as the surface impedance seen by an EM wave normally incident upon a conductor [14]. Using this result, if $t$ is much smaller than the wavelength of submillimeter radiation an inductor with a value of $\mu_{0} t$ can be used to represent the impedance due to magnetic field energy stored between the sheets. This leads to a simple equivalent circuit model for two zero-thickness sheets separated by the distance $t$ [13]. Equating the input impedance $Z_{i n}$ of this equivalent circuit to the actual surface impedance $Z_{s}$ of the finite-thickness superconducting film allows us to solve for the required impedance $Z_{x}$ of the zero-thickness sheets:

$$
\frac{Z_{x}}{Z_{s}}=1-\frac{t}{2 \lambda_{L} \operatorname{coth}\left(t / \lambda_{L}\right)}+\sqrt{1+\frac{t^{2}}{4 \lambda_{L}^{2} \operatorname{coth}^{2}\left(t / \lambda_{L}\right)}}
$$

Dividing $Z_{s}$ and $Z_{x}$ by $\omega$ gives us a surface inductance $\left(L_{s}\right)$ of $88.5 \mathrm{fH} / \mathrm{square}$ and a modified surface inductance $\left(L_{x}\right)$ of 116.6 $\mathrm{fH} / \mathrm{square}$. The above changes in geometry and material properties were incorporated in the SONNET simulations done earlier with single perfectly conducting sheets. The dimensions of the bandpass filter layout were re-optimized to give the corrected bandpasses (Fig. 5). The optical image of the filter is shown in Fig. 4, and the corresponding dimensions are given in Table IV.

\section{MEASUREMENTS AND Results}

Fig. 5 shows the predicted and measured responses of the bandpass filters. The measurement was performed using a Fourier Transform Spectrometer (FTS). Details of the FTS construction are given in [15]. There is reasonable agreement
TABLE 4

X, Y COORDINATES OF THE POINTS IN FIG. 4

\begin{tabular}{rrrrr}
\hline & BPF1 & BPF2 & BPF3 & BPF4 \\
\hline 1 & $148.0,74.0$ & $145.5,74.0$ & $145.5,74.0$ & $145.5,74.0$ \\
2 & $8.5,23.0$ & $8.5,23.0$ & $8.5,23.0$ & $8.5,23.0$ \\
3 & $55.0,23.0$ & $57.0,23.0$ & $57.0,23.0$ & $57.0,23.0$ \\
4 & $106.0,23.0$ & $105.5,23.0$ & $105.5,23.0$ & $105.5,23.0$ \\
5 & $41.5,51.0$ & $39.5,51.0$ & $39.5,51.0$ & $39.5,51.0$ \\
6 & $92.5,51.0$ & $88.0,51.0$ & $88.0,51.0$ & $88.0,51.0$ \\
7 & $139.0,51.0$ & $136.5,51.0$ & $136.5,51.0$ & $136.5,51.0$ \\
8 & $10.5,25.5$ & $12.5,29.5$ & $12.5,29.5$ & $12.5,30.0$ \\
9 & $58.0,25.5$ & $61.0,29.5$ & $61.0,29.5$ & $61.0,30.5$ \\
10 & $108.0,25.5$ & $109.5,29.5$ & $109.5,29.5$ & $109.5,30.0$ \\
11 & $15.5,30.5$ & $15.0,32.5$ & $16.0,34.0$ & $19.5,34.5$ \\
12 & $28.0,30.5$ & $28.0,32.5$ & $27.5,34.0$ & $27.5,34.5$ \\
13 & $60.5,29.5$ & $64.0,32.0$ & $64.0,34.0$ & $67.5,34.5$ \\
14 & $76.0,29.5$ & $76.5,32.0$ & $76.0,34.0$ & $76.0,34.5$ \\
15 & $113.0,30.5$ & $112.0,32.5$ & $113.0,34.0$ & $116.5,34.5$ \\
16 & $125.5,30.5$ & $125.0,32.5$ & $124.5,34.0$ & $124.5,34.5$ \\
17 & $43.0,25.5$ & $43.0,27.0$ & $43.0,28.5$ & $43.5,32.5$ \\
18 & $94.0,25.5$ & $91.5,27.5$ & $91.0,29.0$ & $92.0,32.0$ \\
19 & $0.0,36.0$ & $0.0,36.0$ & $0.0,36.0$ & $0.0,36.0$ \\
\hline & & & &
\end{tabular}

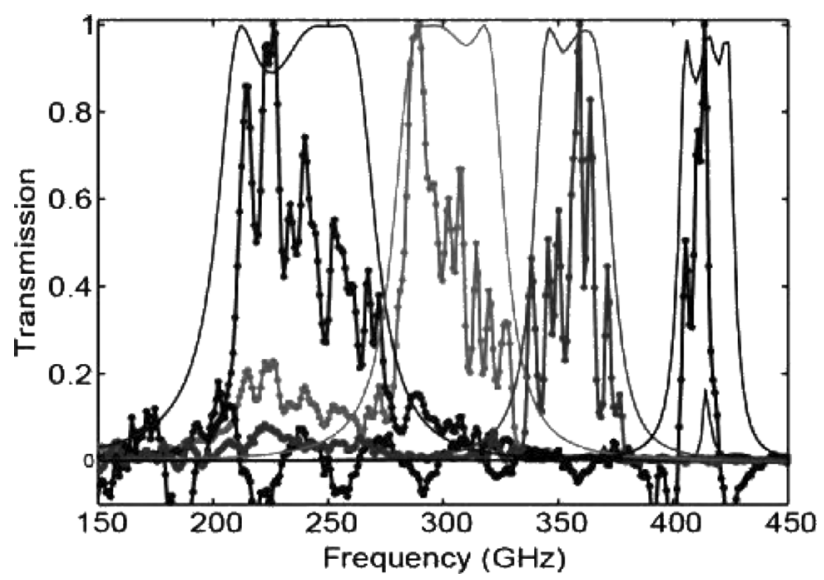

Fig. 5. Normalized measured and predicted bandpass filter responses. Lines with dots are the actual data measured using a Fourier Transform Spectrometer normalized by the maximum transmission value for that filter. The lines without dots are the normalized response predicted using SONNET simulations.

between simulated and measured BPF responses for all 4 bandpass filters, especially considering the numerous factors that affect the measurement other than the filter response, such as the optical transmission of the cryostat window and infrared-blocking filters, the response of the antenna and its combining network, etc. Note that the upper and the lower cutoff frequencies are quite close to their design values. The filters also have sharp turn-ons and turn-offs, and the designed bandwidths match the measured values closely. The fringing seen in the passband of the filter response may be occurring due to the standing waves in the optics (see Fig. 6).

We further confirm that the fringing is not a characteristic of the filter passband by comparing the passband response of a pixel which does not have an inline filter between the antenna and the detector (Fig. 7). Fringing is seen in the response of the bare pixel as well, and the fringe maxima and minima are located at the same frequencies in the response of the pixels containing the filters and ones without them. Furthermore, the electrical delays implied by the rapid fringing are much too long to 


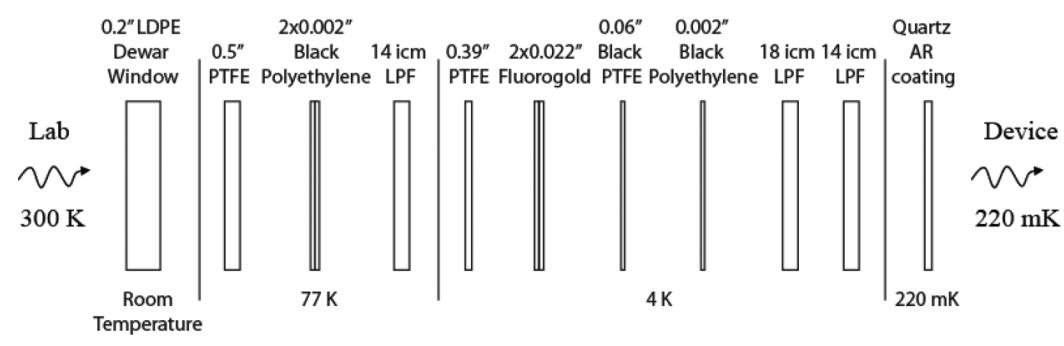

Fig. 6. The optical chain mounted on the dewar windows with temperatures and thicknesses of various IR blockers and metal-mesh filters.

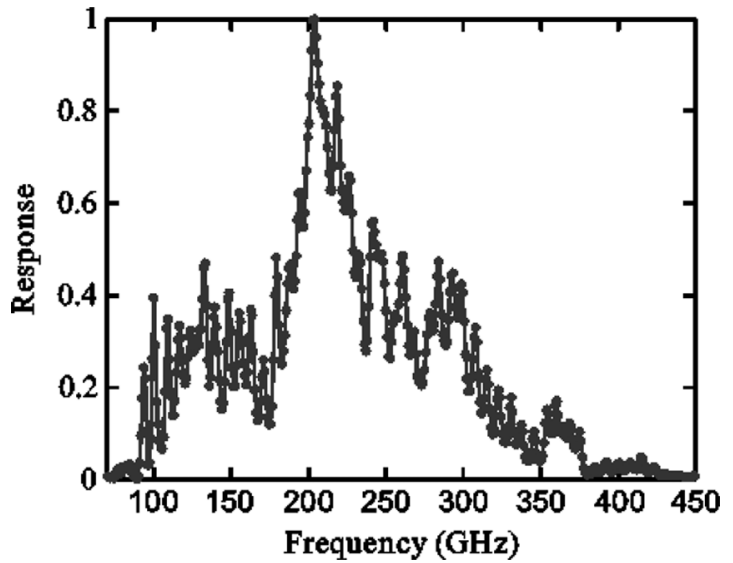

Fig. 7. Normalized measured response of pixel with no inline bandpass filter between the antenna and the detector. The measurement was performed using an FTS. The response turns on at $90 \mathrm{GHz}$ and turns off again at $420 \mathrm{GHz}$.

be explained by the physical length of the filter. The exact source of the passband fringing is currently under investigation. Fig. 7 also shows that the response of the pixel without a bandpass filter is limited by the detector on the low-frequency side. Since energy gap for superconducting aluminum is $90 \mathrm{GHz}$, on-chip microwave kinetic inductance photon detector ([9], [16], [17]) is unresponsive to radiation below $90 \mathrm{GHz}$. On the high-frequency side, the FTS response is limited by the two $420 \mathrm{GHz}(14 \mathrm{icm})$ filters in the optical chain (Fig. 6). In the absence of these filters it would be limited by the high-frequency cutoff frequency of the multi-slot antenna [9] — beyond the niobium bandgap at 700 $\mathrm{GHz}$, the microstrip combining network used for the antenna would become lossy and reduce pixel responsivity drastically. The simulations predict that the filters should show a low-level transmission peak (at a level around $-20 \mathrm{~dB}$ ), possibly their second harmonic due to inductor self-resonance. However, this effect is not clearly visible over the out of band baseline fluctuations in the measured filter responses.

\section{Multi-Color Pixel Design}

One of the primary advantages of fabricating an on-chip bandpass filter is the ability to make multi-color pixels. This can be done by connecting two (or more) filters of different frequency passbands in parallel to the microstrip coming from a single broadband antenna using very short microstrip lines (see Section IV-B). The output microstrips from each of these filters couples power to different detectors allowing each pixel to see multiple bands, using a single broad-band antenna and multiple BPFs and detectors.

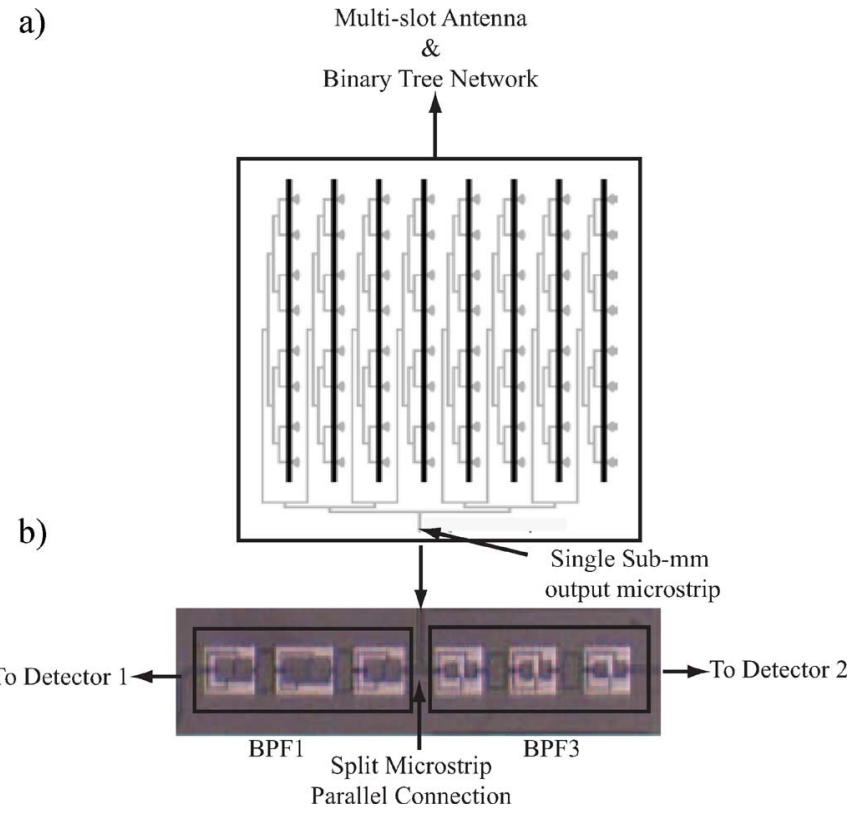

Fig. 8. (a) A schematic of the layout for a two-color pixel showing a single broadband, multi-slot antenna, single microstrip which splits in two and connects to BPF1 and BPF3 in parallel. The outputs of these bandpass filters are connected to different MKIDs made using resonators of different resonance frequency, and readout using a single feedline.

\section{A. Two-Color Design and Results}

Fig. 8 shows the layout for a two-color pixel. For proper operation of the multi-color pixel it is essential that the filters connected in parallel present each other with high impedance outside their bands and don't load each other [9]. We have tested two different two-color pixels, with passband of the first type of pixel defined by BPFs 1 and 2 and that of the second type of pixel by BPFs 1 and 3. Results of the response of the first type of pixel are plotted in Fig. 9. It shows that the response of filters connected in parallel is close to the predicted response of single filters seen in Fig. 5. The overplotted atmospheric transmission spectrum shows that the filter passbands lie well within the transmission windows and away from the water lines. A 16 pixel, two color camera made using pixels has been used for astronomical observation at Caltech Submillimeter Observatory (CSO) [9] to demonstrate proof of principle of operation.

\section{B. Four-Color Design}

The four-color pixel is an extension of the two-color pixel design and can be constructed by connecting four detectors to 


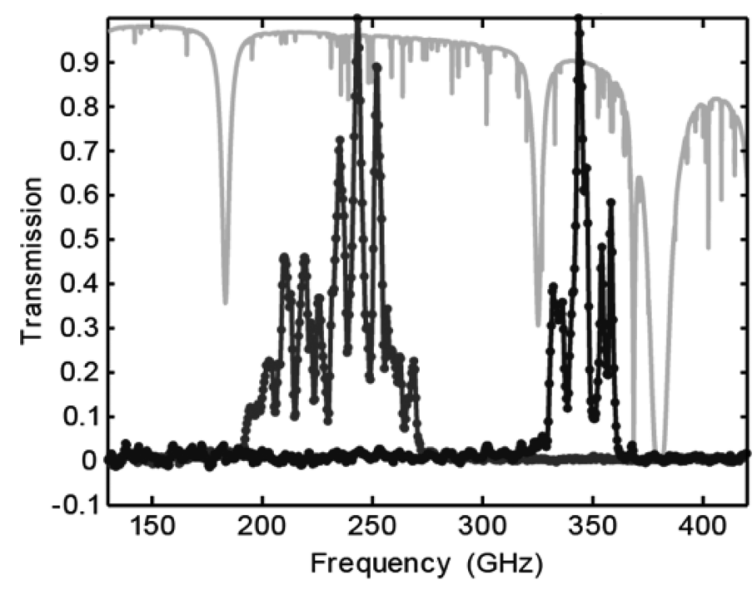

Fig. 9. FTS response of the two-color pixel with the atmospheric transmission spectrum of the Earth's atmosphere overlaid. Filter responses correspond to BPFs $1(209-265 \mathrm{GHz})$ and $3(335-361 \mathrm{GHz})$, respectively. The atmospheric transmission (Grey) as a function of frequency for $0.5 \mathrm{~mm}$ precipitable water vapor plotted using AT (http://www.submillimeter.caltech.edu/cso/weather/atplot.shtml).

a broadband antenna in parallel through four different bandpass filters. It is important to note that although the filters were designed to have high input impedance out of band, sections of transmission lines can act as impedance transformers. If the lengths of the microstrips connecting filter inputs become a significant fraction of $\lambda / 4$, they transform the high input impedances of the filters downwards. At the frequency that this happens, the filter with low input impedance will load the other filters, resulting in passband distortions. Hence, it is essential that the connecting transmission line lengths be much less than $\lambda / 4$, where $\lambda$ is the wavelength at the higher cut off frequency of BPF4. This will also require close geometric placement of filters; however, mutual coupling of the inductors in adjacent filters should be avoided. Details of the network for four-color operation are elaborated in [9].

\section{CONClusions}

We have designed and tested on-chip, lumped-element bandpass filters with passbands corresponding to the atmospheric windows from $209-274 \mathrm{GHz}, 265-315 \mathrm{GHz}, 335-361 \mathrm{GHz}$, and 397-416 GHz. The performance of the filters is well predicted by computer simulations done using SONNET, implying that the fabrication process matches the estimated value for the thickness of $\mathrm{SiO}_{2}$ dielectric $(t=400 \mathrm{~nm})$ and its dielectric constant value $\left(\varepsilon_{d}=4\right)$ reasonably well. Further, we have fabricated pixels with a single antenna coupled to multiple bandpass filters and detectors and demonstrated their two-color operation. Our measurements show no effects due to interaction between the bandpass filters connected to the same input microstrip. The next step is to integrate all the filters into a single pixel and to construct a four color pixel.

\section{REFERENCES}

[1] P. A. R. Ade, G. Pisano, C. Tucker, and S. Weaver, "A review of metal mesh filters," in Proceedings of the SPIE-Millimeter and Submillimeter Detectors and Instrumentation for Astronomy III, J. Zmuidzinas, W. S. Holland, S. Withington, and W. D. Duncan, Eds., Jul. 2006, vol. 6275 , p. $62750 \mathrm{U}$ (2006).

[2] J. Mees, M. Nahum, and P. L. Richards, "New designs for antennacoupled superconducting bolometers," Applied Physics Letters, vol. 59, pp. 2329-2331, Oct. 1991.

[3] R. F. Silverberg, B. Campano, T. C. Chen, E. Cheng, D. A. Cottingham, T. M. Crawford, T. Downes, F. M. Finkbeiner, D. J. Fixsen, D. Logan, S. S. Meyer, T. Perera, E. H. Sharp, and G. W. Wilson, "A bolometer array for the spectral energy distribution (SPEED) camera," Proceedings of the SPIE, vol. 5498, 2004.

[4] S. Kumar, "Submillimeter Wave Camera Using a Novel Photon Detector Technology," Ph.D. Dissertation, Caltech, Pasadena, 2007.

[5] M. J. Myers, W. Holzapfel, A. T. Lee, R. O'Brient, P. L. Richards, H. T. Tran, P. Ade, G. Engargiola, A. Smith, and H. Spieler, "An antenna-coupled bolometer with an integrated microstrip bandpass filter," Applied Physics Letters, vol. 86, no. 11, p. 114103, 2005 [Online]. Available: http://link.aip.org/link/?APL/86/114103/1

[6] D. M. Pozar, Microwave Engineering. Hoboken, NJ: John Wiley and Sons Inc., 1998.

[7] R. E. Collins, Foundations of Microwave Engineering. Piscataway, NJ: IEEE Press, 2001.

[8] Agilent Advanced Design System [Online]. Available: http://eesof.tm. agilent.com/products/ads $\backslash$ main.html

[9] S. S. Mohan, M. del Mar Hershenson, S. P. Boyd, and T. H. Lee, "Simple accurate expressions for planar spiral inductances," IEEE Journal of Solid-State Circuits, vol. 34, Oct. 1999.

[10] Sonnet Software Inc. [Online]. Available: http://www.sonnetusa.com/ [Online]. Available:

[11] D. C. Mattis and J. Bardeen, "Theory of the anomalous skin effect in normal and superconducting metals," Physical Review, vol. 111, no. 2, pp. 412-417, 1958.

[12] A. Vayonakis, H. Leduc, C. Luo, and J. Zmuidzinas, Precision measurements of the millimeter wave properties of superconducting thin-film microstrip lines 2008, Internal Communication.

[13] A. Kerr, Surface impedance of superconductors and normal conductors in EM simulators 1999 [Online]. Available: http://www.alma.nrao.edu/ memos/

[14] R. Matick, Transmission Lines for Digital and Communication Networks. New York: McGraw-Hill, 1969.

[15] M. Bin, D. J. Benford, M. C. Gaidis, T. Buttgenbach, J. Zmuidzinas, E. Serabyn, and T. Phillips, "A large throughput high resolution Fourier Transform Spectrometer for submillimeter applications," International Journal of Infrared and Millimeter Waves, vol. 20, pp. 383-400, 1999.

[16] B. A. Mazin, "Microwave Kinetic Inductance Detectors," Ph.D. Dissertation, , Caltech, Pasadena, 2004.

[17] P. K. Day, H. G. LeDuc, B. A. Mazin, A. Vayonakis, and J. Zmuidz inas, "A broadband superconducting detector suitable for use in large arrays," Nature, vol. 425, pp. 817-821, Oct. 2003. 